\title{
BEHAVIOUR OF SWEEP LINE WITH HEAVY BALL*
}

\author{
Otohiko Suzuki** \\ (Received March 16, 1965)
}

It is usual, in Japanese ground trawling, to connect a sweep line and a tow rope by a length of heavy chain. This chain is commonly considered to serve for the increase of the sweeping efficiency of gear as well as the prevention of departure of sweep line from the sea bottom.

In order to get informations about the effect of chain on sweep line, a fundamental study is developed in a simple case that a heavy ball is attached to a uniform rope.

\section{Theoretical Consideration}

A uniform flexible rope A-B-C is laid at first on a horizontal sand plane in such a form that its parts $\overline{\mathrm{AB}}$ and $\overline{\mathrm{BC}}$ are bent at a point $\mathrm{B}$ making a right angle, and a heavy ball is attached to the rope at a distance $L$ along the line $\overline{\mathrm{AB}}$ measured

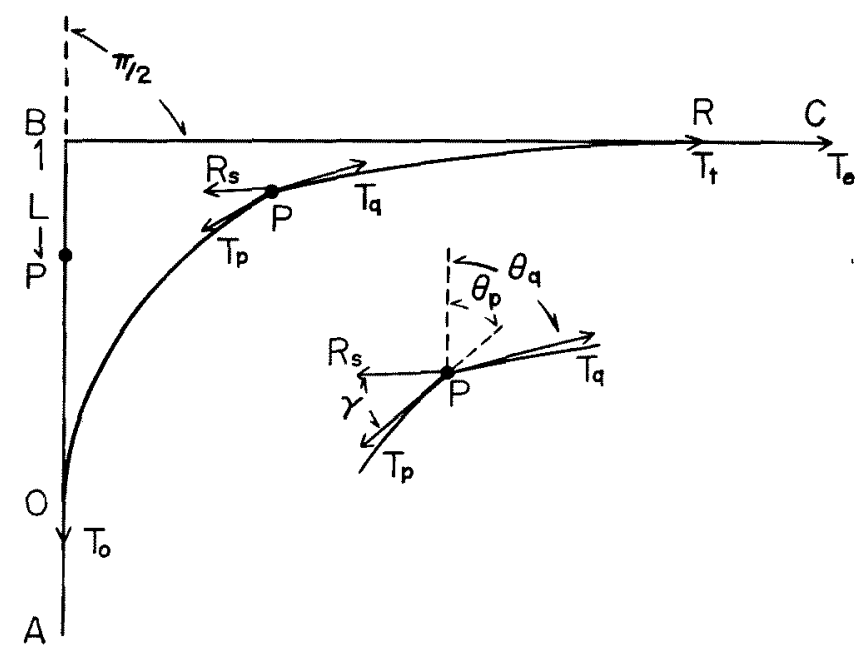

Fig. 1. Form of sweep line fitted with heavy ball in layout and in motion.

from the point $B$ (Fig. 1). If the free end $C$ of rope is pulled slowly to a definite direction $\overline{\mathrm{BC}}$, there appears a sharp bend at a point $\mathrm{P}$ along the rope to which the ball is attached. With increase of towed distance of rope end $\mathrm{C}$, the bend will

* Studies on the kinematic behaviour of the ground rope of the trawl net-IX.

** Department of Fisheries, Kyoto University, Maizuru (鈴木乙彦, 京都大学農学部本産学教室) 
disappear and the pattern of rope will become one smooth curve, since the tension acting on rope at the point $P$ becomes sufficiently large as compared with the resistance of the ball.

Referring to Fig. 1, take the $y$-axis along the direction of the part $\overline{\mathrm{AB}}$ of originally laid rope and choose the origin at the point $O$ where the curve $\widehat{O P}$ comes in contact with the $y$-axis. Denote by $R_{s}$ the frictional resistance of the ball, and let $T_{p}$ and $T_{q}$ be the tensions acting on the respective parts of rope $\widehat{O P}$ and $\overparen{P R}$ at the point $P$. $\theta_{p}$ and $\theta_{q}$ be the angles of the directions of tension $T_{p}$ and $T_{q}$ relative to the $y$-axis, respectively, and $\gamma$ the angle between the directions of $T_{p}$ and $R_{s}$. Resolution of these forces into the direcitons of $T_{p}$ and its normal gives,

$$
\begin{gathered}
T_{p}+R_{s} \cos \gamma=T_{q} \cos \left(\theta_{q}-\theta_{p}\right), \\
R_{s} \sin \gamma=T_{q} \sin \left(\theta_{q}-\theta_{p}\right) .
\end{gathered}
$$

Separately from the above circumstances, we consider a form assumed by a uniform rope, omitted the ball. The originally laid form of rope is deformed to a smooth curve by towing the free end of rope, as mentioned in the references. ${ }^{1.2)}$ On this smooth curve $\overparen{O R}$ of rope carrying no ball, we now choose points $\mathrm{P}$ and $\mathrm{Q}$ at the respective points where the directions of rope make angles $\theta_{p}$ and $\theta_{q}$ relative to the $y$-axis. Here, imagine that the tensions acting on rope at the points $\mathrm{P}$ and $\mathrm{Q}$, at an instant of towing, just become $T_{p}$ and $T_{q}$, respectively. The form assumed by the parts of rope, $\widehat{O P}$ and $\overparen{Q R}$, thus obtained should be geometrically congruent with that of the parts of rope, $\widehat{O P}$ and $\overparen{P R}$, shown in Fig. 1, since the mechanical relations by which the form of rope is governed must be the same as in the references. ${ }^{1,2}$ In other words, the form of rope in Fig. 1 may be regarded as that obtained by the following procedure: cut off the part $\widehat{\mathrm{QQ}}$ from the whole curve $\widehat{\mathrm{OR}}$ and remove the curve $\widehat{\mathrm{QR}}$ keeping the angle of the direction of $T_{q}$ relative to the $y$-axis to $\theta_{q}$ in such a manner that the point $\mathrm{Q}$ just comes to the point $\mathrm{P}$ of the curve $\widehat{O P}$. Accordingly, we have the following relation, in accordance with the equation (3) of the reference:1)

$$
\frac{T_{q}}{T_{p}}=e^{\kappa\left(\theta \theta_{q}-\theta_{p}\right)},
$$

where $\kappa=K_{t} / K_{n}$, denoting by $K_{t}$ and $K_{n}$ the tangential and the normal component of frictional force per unit length of rope.

Substitution of the equation (3) into the relations (1) and (2) gives

$$
\begin{aligned}
& \cot \gamma=\cot \left(\theta_{q}-\theta_{p}\right)-e^{-\kappa\left(\theta_{q}-\theta_{p}\right)} \operatorname{cosec}\left(\theta_{q}-\theta_{p}\right), \\
& \left(R_{s} / T_{p}\right)^{2}=e^{2 \kappa\left(\theta_{q}-\theta_{p}\right)}-2 e^{\kappa\left(\theta_{q}-\theta_{p}\right)} \cos \left(\theta_{q}-\theta_{p}\right)+1 .
\end{aligned}
$$

Moreover, dedote by $s_{1}$ the arc length of the curve $\widehat{\mathrm{OP}}, x_{1}$ and $y_{1}$ its projections to the $x$ - and the $y$-axis, and let $s_{2}, x_{2}$ and $y_{2}$ be the corresponding quantities of the curve $\overparen{P R}$. Then, the following relations may be obtained by the reference to Fig. 1, 


$$
\begin{gathered}
X+Y-S=l, \\
Y-s_{1}=L,
\end{gathered}
$$

in which $S=s_{1}+s_{2}, X=x_{1}+x_{2}, Y=y_{1}+y_{2}$ and $l$ is the towed distance of the rope end C. In accordance with the equations (4), (5) and (6) of the reference, ${ }^{1)} S, X, Y$ and $s_{1}$ are expressed as:

$$
\begin{aligned}
& S=\frac{T_{p}}{K_{n}} \frac{1}{\kappa}\left(e^{\kappa\left(\pi / 2-\theta_{p}\right)}-e^{\kappa\left(\theta_{q}-\theta_{p}\right\}}-e^{\kappa \theta_{p}}+1\right), \\
& X=\frac{T_{p}}{K_{n}} \frac{1}{\kappa^{2}+1}\left[\kappa e^{\kappa\left(\pi / 2-\theta_{p}\right)}-e^{\kappa\left(\theta_{q}-\theta_{p}\right)}\left(\kappa \sin \theta_{q}-\cos \theta_{q}\right)+\left(\kappa \sin \theta_{p}-\cos \theta_{p}\right)+e^{-\kappa \theta} p\right], \\
& Y=\frac{T_{p}}{K_{n}} \frac{1}{\kappa^{2}+1}\left[e^{\kappa\left(\pi / 2-\theta_{p}\right)}-e^{\kappa\left(\theta_{q}-\theta_{p}\right)}\left(\kappa \cos \theta_{q}+\sin \theta_{q}\right)+\left(\kappa \cos \theta_{p}+\sin \theta_{p}\right)-\kappa e^{-\kappa \theta} p\right], \\
& s_{1}=\frac{T_{p}}{K_{n}} \frac{1}{\kappa}\left(1-e^{-\kappa \theta_{p}}\right) .
\end{aligned}
$$

It is seen at once from the equations (4) and (5) that the value of $\gamma$ and the ratio $R_{s} / T_{p}$ are functions of $\left(\theta_{q}-\theta_{p}\right)$, if the value of $\kappa$ is known. Division of the

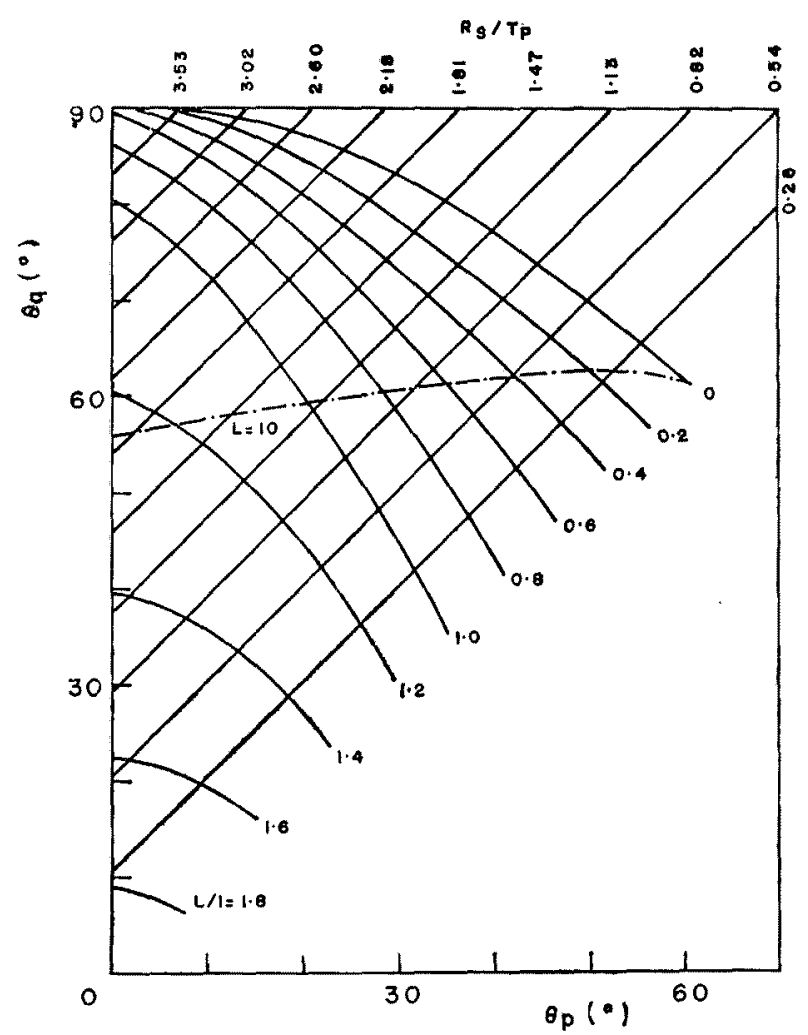

Fig. 2. Isopleths of $R_{8} / T_{p}$ and $L / l$ for $\kappa=0.85$. Broken line shows relation of $\theta_{q}$ versus $\theta_{p}$ for $L=10 \mathrm{~cm}$. and $R_{s}=2.11 \mathrm{gr}$. 
equation (7) by (6), in consideration of the equations (8) $\sim(11)$, eliminates $T_{p} / K_{n}$ and gives the ratio $L / l$ as a function of two variables $\theta_{p}$ and $\theta_{q}$. In Fig. 2 is shown the isopleth of $L / l$ together with that of $R_{s} / T_{p}$, letting $\kappa=0.85$. This value of $\kappa$ corresponds to that given from experimental conditions, as will be seen later. From the figure, the ratio $R_{s} / T_{p}$ is determined as a function of $\theta_{p}$ for a definite value of $\theta_{q}$. Combining the equation (7) and the relation between $R_{s} / T_{p}$ and $\theta_{p}$ for arbitrary $\theta_{q}$ and giving the value of $R_{s}$, we can obtain the relation of $\theta_{g}$ versus $\theta_{p}$ for a given value of $L(\neq 0)$ by trial and error. In a special case of $L=0$, it holds that $L / l=0$. In consequance, the curve $L / l=0$ determines the values of $R_{s} / T_{p}$ and $\theta_{q}$ as functions of $\theta_{p}$. Thus, we can obtain the relations of $R_{s} / T_{p}$ versus $\theta_{p}$ and $\theta_{q}$ versus $\theta_{p}$ for an arbitrary value of $L$. In the figure the curves of $L=0$, and $10 \mathrm{~cm}$. are illustrated: the curve of $L=10 \mathrm{~cm}$. is calculated with substitution of $R_{s}=2.11 \mathrm{gr}$. which is given by experimental condition.

From the data given above the values of $S, X$ and $Y$, characteristic for the pattern of rope, can be calculated as functions of $\theta_{p}$. Since, moreover, the towed distance $l$ can also be obtained as a function of $\theta_{p}$ in terms of the equation (6), $S$, $X$ and $Y$ are reversely expressed as functions of $l$. The tension $T_{t}$ acting on rope at the point $R$ is

$$
T_{t}=T_{p} e^{\kappa\left(\pi / 2-\theta_{p}\right)},
$$

and the tension $T_{e}\left[=T_{t}+K_{t} \times \overline{R C}\right]$ acting on rope at the end $C$ is also calculated as a function of $l$, since the linear part $R C$ is calculated as a function of $l$. Thus, we can predict how the form and tension of rope under consideration vary with the towed distance.

\section{Experimental}

An arrangement of experimental apparatus employed was illustrated schematically in the reference. ${ }^{3)}$ A uniform nylon twine of $3 \mathrm{~mm}$. in diameter, whose values of $K_{t}$ and $K_{n}$ are 0.0440 and $0.0517 \mathrm{gr} . / \mathrm{cm}$. respectively, was laid on a horizontal plane of dry sand in such a form as shown in Fig. 1, letting $\overline{A B}=90 \mathrm{~cm}$. and $B C=100 \mathrm{~cm}$. A small lead ball whose frictional resistance is $2.11 \mathrm{gr}$. was attached to the twine at the point $B(L=0)$ in one of duplicate experiments, and, in the other, at the point of $L=10 \mathrm{~cm}$. Then the free end $C$ of the twine was towed to the direction $\overline{B C}$ as far as $30 \mathrm{~cm}$. in the speed range of 0.7 to $1.0 \mathrm{~cm} . / \mathrm{sec}$. At a regular interval $5 \mathrm{~cm}$. of towing, the towing force was measured and simultaneously the form of twine was photographed from the top.

\section{Results and Discussion}

In the case of $L=0 \mathrm{~cm}$, the ball starts to move as soon as the twine end $C$ is pulled. Accordingly, as mentioned in the theoretical, the values of $S, X$ and $Y$ are 
determined as functions of $l$ throughout the whole course of towing.

In the case of $L=10 \mathrm{~cm}$., however, the ball does not start to move so immediately. The behaviour of twine can be divided into three stages as follows: (1) from the beginning of towing to the propagation of the deformation of rope into the point $P$, (2) thereafter to the moment when the ball starts to move, and (3) after the time when the ball has moved. The data for the behaviour in the first stage are available from the references, ${ }^{1,2)}$ from which $l$ is known to be about $5 \mathrm{~cm}$. when the deformation of rope propagates to the point P. Moreover, Fig. 2 gives the value of $\theta_{p}\left(\fallingdotseq 56^{\circ}\right)$ at the moment when the ball starts to move. The value of $l$, at this instant, is calculated to be about $8 \mathrm{~cm}$. In the second stage that $5<l<8$, i.e. $0<\theta_{q}<56^{\circ}$, since the ball retains its initial position, $\theta_{p}=0^{\circ}$ and $y_{2}=10 \mathrm{~cm}$. Hence, the following relation is obtained,

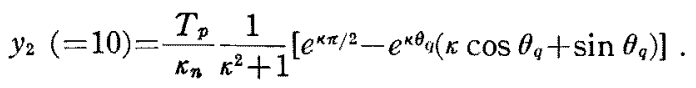

It is seen that the above expression gives the relation of $T_{p}$ and $\theta_{q}$ in $0<\theta_{q}<56^{\circ}$.

By the use of this relation, the values of $s_{2}$ and $x_{2}$, are also calculated as functions of $\theta_{q}$, and, alternatively, are expressed as functions of $l$. In the range $l>8, S, X$

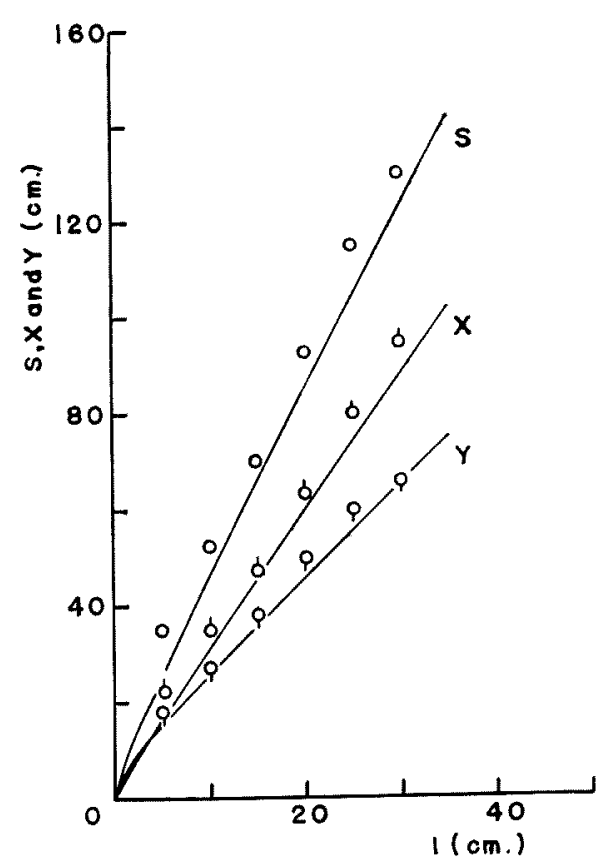

a

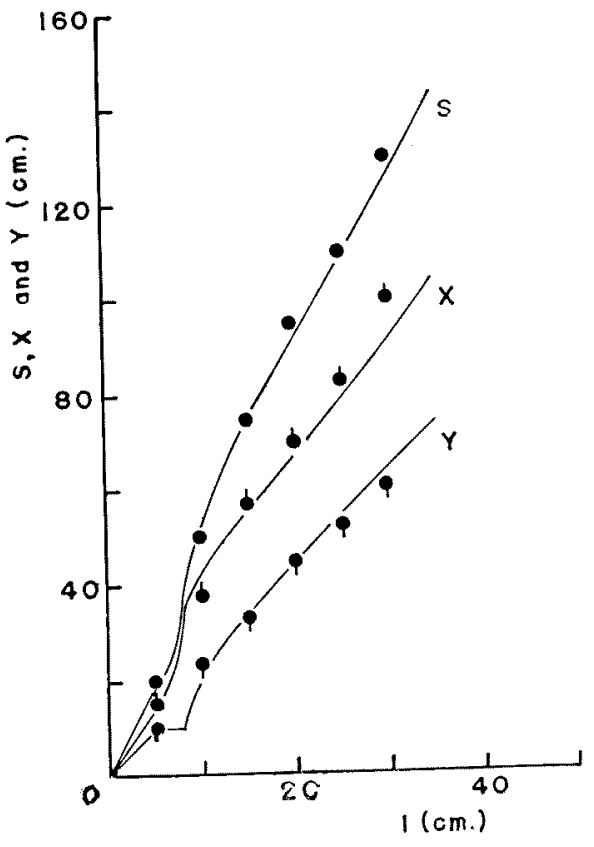

$\mathrm{b}$

Fig. 3-a. Characteristic values, $S, X$ and $Y$, of sweep line plotted against towed distance $l$, for $L=0 \mathrm{~cm}$. $O$ : Experimental; - : Theoretical.

Fig. 3-b. Similar plots as in Fig. 3 -a for $L=10 \mathrm{~cm}$. : Experimental; - : Theoretical 
and $Y$ are determined with the aid of Fig. 2 and the relation (6). In Figs. 3-a and 3-b are plotted the values of $S, X$ and $Y$ as functions of $l$ in the respective case of $L=0$ and $10 \mathrm{~cm}$., together with their observed ones.

In Fig. 4 the calculated and the observed values of $T_{e}$ are plotted against $l$.

The author is indebted to Prof. T. Kawakamr for his helpful criticism of the manuscript.

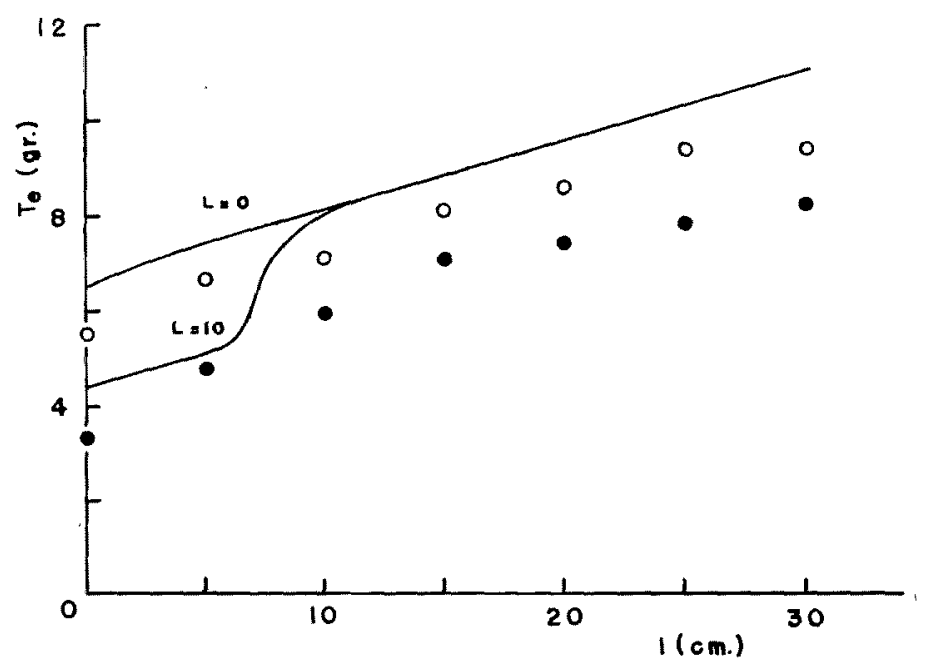

Fig. 4. Tension $T_{e}$ as a function of towed distance $l$. $\bigcirc$, : Experimental values for $L=0$ and $10 \mathrm{~cm}$., respectively; --: Theoretical.

\section{References}

1) SuzukI, O.: Bull. Jap. Soc. Sei. Fish., 28, 985-987 (1962).

2) : ibid., 28, 988-991 (1962).

3) KaWAKaMI, T. and O. SUZUKI: ibid., 25, 413-416 (1959). 